\title{
Implications of prognostic pessimism in patients with chronic obstructive pulmonary disease (COPD) or asthma admitted to intensive care in the UK within the COPD and asthma outcome study (CAOS): multicentre observational cohort study
}

\author{
Martin J Wildman, consultant chest physician, ${ }^{1}$ Colin Sanderson, reader in health services research, ${ }^{2}$ \\ Jayne Groves, research nurse, ${ }^{3}$ Barnaby C Reeves, reader in epidemiology, ${ }^{2}$ Jon Ayres, professor, ${ }^{4}$ \\ David Harrison, senior statistician, ${ }^{5}$ Duncan Young, consultant intensivist, ${ }^{6}$ Kathy Rowan, director ${ }^{5}$
}

${ }^{1}$ Northern General Hospital, Sheffield

${ }^{2}$ London School of Hygiene and Tropical Medicine, London

${ }^{3}$ Birmingham Heartlands Hospital, Birmingham

${ }^{4}$ Department of Occupational and Environmental Medicine,

Liberty Safe Work Research

Centre, Aberdeen

${ }^{5}$ Intensive Care National Audit and Research Centre, London

${ }^{6}$ Adult Intensive Care Unit, John Radcliffe Hospital, Oxford

Correspondence to: $M$ Wildman martin.wildman@sth.nhs.uk

doi:10.1136/bmj.39371.524271.55

\section{ABSTRACT}

Objective To determine whether clinicians' prognoses in patients with severe acute exacerbations of obstructive lung disease admitted to intensive care match observed outcomes in terms of survival.

Design Prospective cohort study.

Setting 92 intensive care units and three respiratory high dependency units in the United Kingdom.

Participants 832 patients aged 45 years and older with breathlessness, respiratory failure, or change in mental status because of an exacerbation of COPD, asthma, or a combination of the two.

Main outcome measures Outcome predicted by clinicians. Observed survival at 180 days.

Results 517 patients (62\%) survived to 180 days. Clinicians' prognoses were pessimistic, with a mean predicted survival of $49 \%$ at 180 days. For the fifth of patients with the poorest prognosis according to the clinician, the predicted survival rate was $10 \%$ and the actual rate was $40 \%$. Information from a database covering $74 \%$ of intensive care units in the UK suggested no material difference between units that participated and those that did not. Patients recruited were similar to those not recruited in the same units.

Conclusions Because decisions on whether to admit patients with COPD or asthma to intensive care for intubation depend on clinicians' prognoses, some patients who might otherwise survive are probably being denied admission because of unwarranted prognostic pessimism.

\section{INTRODUCTION}

Each year in the United Kingdom, around 30000 deaths are associated with chronic obstructive pulmonary disease (COPD). ${ }^{1}$ Many patients with exacerbations of COPD benefit from assisted ventilation, but for intubation the patient must be admitted to an intensive care unit. COPD accounts for 3\% of such admissions in the UK, with a median stay of 16 days (interquartile range $9-29$ days). ${ }^{2}$
Doctors consider prognosis to be of "paramount importance" in deciding which patients should be admitted to intensive care, ${ }^{3}$ and admission might be refused. ${ }^{4}$ Prognosis, however, can be difficult, ${ }^{56}$ and in an American study doctors' predictions of survival tended to be pessimistic compared with a prognostic model. $^{7}$ If prognoses for patients in the UK with exacerbations of COPD are also unduly pessimistic, some patients with reasonable medium term prognoses might be being denied admission to intensive care for intubation and care.

\section{METHODS}

We invited all intensive care units participating in the UK case mix programme ${ }^{8}$ and three respiratory high dependency units to take part. Eligible patients were those admitted to participating units for breathlessness, respiratory failure, or change in mental status because of an exacerbation of obstructive lung disease. Patients were excluded if they were aged under 45 years or had had surgery within the past 10 days or had been transferred directly from another hospital to the unit. Data were collected for admissions from March 2002 to September 2003 with follow-up to 180 days after admission to intensive care.

On admission, the admitting doctor was asked to estimate the patient's probability of survival to discharge from intensive care or high dependency, to discharge from hospital, and at 180 days after admission. We determined actual survival to 180 days from the general practitioner and confirmed this through the Office for National Statistics. Data were also collected on prognostic variables.

Analyses were carried out using Stata version 9 (Stata Corp, College Station, Tx).

\section{RESULTS}

Of 239 intensive care units in the UK in January 2002, 177 were contributing to the case mix programme. Of 


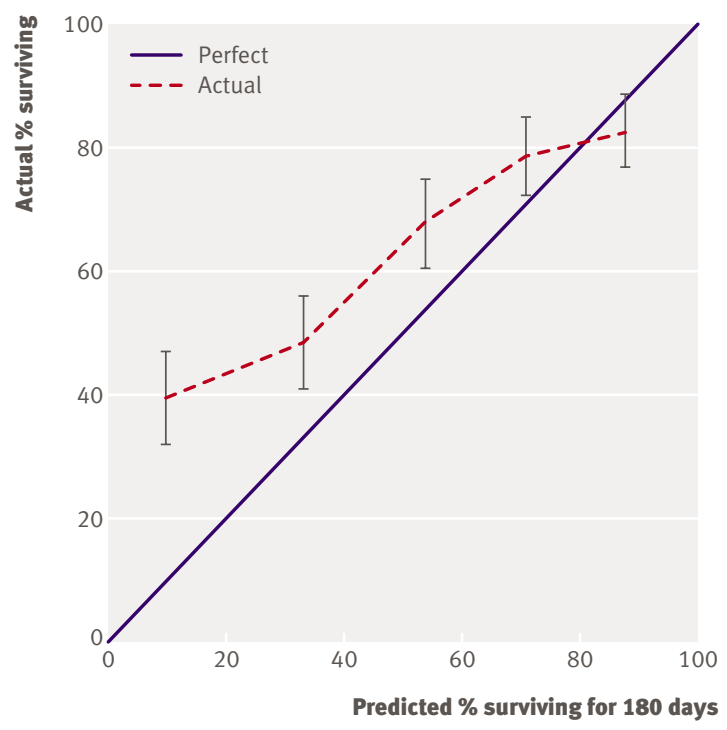

Actual 180 day survival ( $95 \%$ confidence interval) by fifth of predicted survival

these, 92 units participated in our study, together with the three high dependency units. Data from the case mix programme showed that participating and nonparticipating units were similar in type, size, affiliation, overall percentage of admissions potentially eligible for the study (3.9 v 3.2\%), acute physiology scores for $\mathrm{COPD},{ }^{9}$ and survival in intensive care for potentially eligible admissions. We recruited 832 patients (724 from intensive care and 108 from high dependency). In intensive care units taking part in the case mix programme there were no differences between the 648 patients recruited to our study and the 996 not recruited in terms of age ( $\mathrm{z}$ test $\mathrm{P}=0.49)$, acute physiology score $(\mathrm{z}$ test $\mathrm{P}=0.96)$, or survival in intensive care $\left(\chi^{2} 0.77, \mathrm{P}=0.38\right)$.

Survival rates were $80.9 \%$ (95\% confidence interval $78.1 \%$ to $83.5 \%$ ) at discharge from intensive care or high dependency, $70.2 \%(67.0 \%$ to $73.3 \%)$ at discharge from hospital, and $62.1 \%(58.7 \%$ to $65.4 \%)$ at 180 days after admission to intensive care or high dependency. We compared mortality at 180 days predicted by clinicians with the actual 180 day mortality, overall and for different subgroups defined by age, sex, previous function, diagnosis, mean arm circumference, and acute physiology score. We found a tendency towards prognostic pessimism, overall and for most subgroups.

\section{WHAT IS ALREADY KNOWN ON THIS TOPIC}

Simulation studies in the UK have shown that clinicians are pessimistic in predicting survival after admission to intensive care for patients with chronic obstructive pulmonary disease

\section{WHAT THIS STUDY ADDS}

Similar pessimism is observed in clinical practice and has the potential to distort clinical decision making
The evidence for prognostic pessimism was weakest in the following subgroups: age $\geq 75$ years, mean arm circumference $<25 \mathrm{~cm}$, worst quarter for acute physiology score, and chairbound or bedbound before admission to intensive care or high dependency.

Clinician pessimism was particularly marked for the patients in the lower fifth of the distribution of prognosis (figure). In fact, the tenth of patients with the poorest clinician prognosis had a predicted 180 day survival of around $3 \%$ and an actual survival of around $36 \%$.

\section{DISCUSSION}

Clinicians are generally pessimistic about the survival prospects of patients with exacerbations of COPD and have particular problems in identifying those with poor prognosis. Patients might therefore be inappropriately excluded from intensive care and the chance of intubation on the basis of a false prediction of futility. The units and patients recruited to this study seem to be representative of UK practice.

\section{Limitations}

That we recruited only patients admitted to intensive care or high dependency is a clear limitation. The general level of pessimism among those refused admission, however, was likely to have been even greater than in those admitted. We cannot see how it could have been less. Prognostic pessimism was found across a wide range of subgroups by severity and was also found in a US study that was not limited to patients in critical care. ${ }^{8}$

Historically, access to intensive care in the UK has been problematic and it has not always been possible to admit every patient who might benefit. A culture of pessimism might protect clinicians from the cognitive dissonance involved in being unable to intubate patients they knew to have a reasonable prognosis. When renal dialysis was much less available the discussions around withholding it were often accompanied by predictions of futility if it were to be offered. ${ }^{10}$ In the context of triage of patients with COPD, however, this pessimism might be distorting decision making.

We thank all the units that participated in the CAOS study and the intensive care national audit and research centre for its invaluable role in recruiting units and data linkage. We thank Jan Van Der Meulen for advice on the data analysis.

Contributors: MJW, CS, KR, JA, BCR, and DY were involved in the original design of the study. MJW coordinated the study, carried out the initial data analysis, and drafted the paper. JG helped to collect the data. CS carried out additional analyses and drafted the final paper. $\mathrm{KR}$ and $\mathrm{DH}$ helped recruit the units and facilitated data linkage with the case mix programme. MJW is guarantor.

Funding: MJW was funded by an MRC Health Services Research Fellowship. JG was funded by an NHS research and development grant. Competing interests: None declared.

Ethical approval: Multicentre research ethics committees.

Provenance and peer review: Not commissioned; externally peer reviewed.

1 Britton $M$. The burden of COPD in the UK: results from the confronting COPD survey. Respir Med 2003;97(suppl C):S71-9. 
2 Wildman MJ, Harrison DA, Brady ARB, Rowan K. Case mix and outcomes for admissions to UK adult, general critical care units with chronic obstructive pulmonary disease: a secondary analysis of the ICNARC case mix programme database. Critical Care 2005;9(suppl 3):S38-48.

3 Escher M, Perneger TV, Chevrolet I-C. National questionnaire survey on what influences doctors' decisions about admission to intensive care. BMJ 2004;329:425-8

4 Joynt G, Gomersall C, Tan P, Lee A, Cheng C, Wong E. Prospective evaluation of patients refused admission to an intensive care unit: triage, futility and outcome. Intens Care Med 2001;27:1459-65.

5 Pearlman RA. Variability in physician estimates of survival for acute respiratory failure in chronic obstructive pulmonary disease. Chest 1987;91:515-21.

6 Wildman M, O'Dea J, Kostopolou O, Tindall M, Walia S, Khan Z. Variation in intubation decisions for patients with chronic obstructive pulmonary disease in one critical care network. Q J Med 2003;96:583-91.
7 Connors AF Jr, Dawson NV, Thomas C, Harrell FE Jr, Desbiens N, Fulkerson WJ, et al. Outcomes following acute exacerbation of severe chronic obstructive lung disease. The SUPPORT investigators (study to understand prognoses and preferences for outcomes and risks of treatments). Am J Respir Crit Care Med 1996;154:959-67.

8 Harrison DA, Brady AR, Rowan K. Case mix, outcome and length of stay for admissions to adult, general critical care units in England, Wales and Northern Ireland: the intensive care national audit and research centre case mix programme database. Crit Care 2004;8:R99-111.

9 Wildman MJ, Harrison DA, Welch CA, Sanderson C. A New measure of acute physiological derangement for patients with exacerbations of obstructive airways disease: the COPD and Asthma Physiology Score. Respir Med 2007;101:1994-2002.

10 Parsons V, Lock P. Triage and the patient with renal failure. J Med Ethics 1980;6:173-6.

Accepted: 10 September 2007 